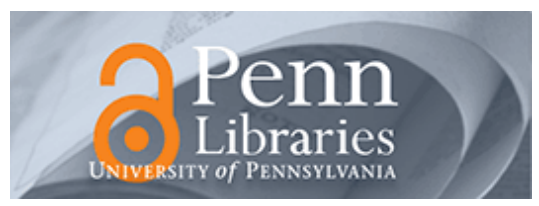

University of Pennsylvania ScholarlyCommons

March 2004

\title{
Numerical Study of Polarization-Dependent Focusing for a Bilayer Planar FSS Reflective Lens at Millimeter Wavelengths
}

\author{
Natalia Bliznyuk \\ University of Pennsylvania \\ Nader Engheta \\ University of Pennsylvania, engheta@ee.upenn.edu
}

Follow this and additional works at: https://repository.upenn.edu/ese_papers

\section{Recommended Citation}

Natalia Bliznyuk and Nader Engheta, "Numerical Study of Polarization-Dependent Focusing for a Bilayer Planar FSS Reflective Lens at Millimeter Wavelengths", . March 2004.

Postprint version. "This is a preprint of an article published in Microwave and Optical Technology Letters, Volume 40, Issue 5, March 5, 2004, pages 361-365."

Publisher URL: http://www3.interscience.wiley.com/cgi-bin/jissue/106601004

This paper is posted at ScholarlyCommons. https://repository.upenn.edu/ese_papers/272

For more information, please contact repository@pobox.upenn.edu. 


\title{
Numerical Study of Polarization-Dependent Focusing for a Bilayer Planar FSS Reflective Lens at Millimeter Wavelengths
}

\begin{abstract}
We analyze numerically the polarization-dependent focusing properties of a planar reflective lens that is formed by two parallel layers of the non-uniform "gangbuster surfaces (GS)" above a ground plane. Since these gangbuster surfaces consist of superdense arrays of thin finite-length parallel metallic wires, the desired phase patterns on such a lens surface are achieved by adjusting lengths of these metallic wires for appropriate polarization components. In our analysis, we use the Method of Moment (MoM) and the transmission lines analogy, along with the surface equivalence theorem, to estimate the field intensity in the focal plane.
\end{abstract}

\section{Keywords}

frequency-selective surface, millimeter-wave, method of moments, focal plane, lens

\section{Comments}

Postprint version. "This is a preprint of an article published in Microwave and Optical Technology Letters, Volume 40, Issue 5, March 5, 2004, pages 361-365."

Publisher URL: http://www3.interscience.wiley.com/cgi-bin/jissue/106601004 


\title{
Numerical Study of Polarization-Dependent Focusing for a Bilayer Planar FSS Reflective Lens at Millimeter Wavelengths
}

\author{
Natalia Bliznyuk and Nader Engheta \\ University of Pennsylvania \\ Department of Electrical and Systems Engineering, \\ Philadelphia, Pennsylvania 19104-6390, U.S.A. \\ E-mail:natali@ee.upenn.edu,engheta@ee.upenn.edu \\ URL: http://www.ee.upenn.edu/ engheta/
}

\begin{abstract}
We analyze numerically the polarization-dependent focusing properties of a planar reflective lens that is formed by two parallel layers of the non-uniform "gangbuster surfaces (GS)" above a ground plane. Since these gangbuster surfaces consist of superdense arrays of thin finite-length parallel metallic wires, the desired phase patterns on such a lens surface are achieved by adjusting lengths of these metallic wires for appropriate polarization components. In our analysis, we use the Method of Moment $(\mathrm{MoM})$ and the transmission lines analogy, along with the surface equivalence theorem, to estimate the field intensity in the focal plane.
\end{abstract}




\section{Introduction}

It is well known that passive millimeter-wave (MMW) imaging systems can provide useful imaging tools in certain atmospheric conditions, e.g., fog and clouds, with advantages over the standard visible imagery mainly due to the lower attenuation of millimeter waves in such conditions in comparison to the visible and infrared (IR) domains. But the design of such MMW imaging systems presents certain challenges, since the use of conventional lens designs for the millimeter-wave regimes would result in relatively bulky and heavy optics. Thus, novel innovation and ideas are needed in order to design MMW lensing systems that are relatively small, thin and light weight. In the millimeter wave band, there have been several kinds of lenses considered so far. Among those, one can mention the refractive (e.g., [1-3]) and diffractive lenses (e.g., [46], and also focusing systems based on the use of frequency-selective surfaces (FSS) in antennas and in reflectarray applications (e.g., [7-11]).

As is known, the fundamental idea behind most of the focusing systems is indeed reshaping of the phase front of the incident wave such that the new phase distribution would cause convergence of the wave into a certain desired focal point/region. In our current problem, we intend to design a flat, thin lens that reflects the incident wave completely, but upon such reflection, we desire to reshape the phase of the reflected wave along the lens surface such that it focuses the wave at a specific focal point. In order to achieve this, we use a class of frequency-selective surfaces (FSS), known as the "gangbuster" surfaces (GS) [12]. By having this surface above a ground plane, one can achieve a complete reflection of the incident wave, and furthermore, by judiciously designing the elements of this GS, one can achieve the desired phase distribution for the 
reflected wave. In other words, by changing local geometry of this FSS, e.g., size of the unit element, one can control phase of the reflected field. If the range of such phase shift is large enough and spans almost $2 \pi$, such a reflective lens can be used in the imaging systems. Moreover, since the wave interaction with the GS is strongly dependent on the wave's polarization, we use two parallel GS's with orthogonally directed wire elements, as shown in Fig. 1, in order to independently tailor focusing properties of each orthogonal polarization.

In general, in the past some of the focusing properties of tapered FSS have been investigated experimentally in [e.g., 8] and theoretically in various studies devoted to reflectarrays [e.g., 6-11]. Therefore, FSS can be a good candidate for the design of such lenses, with flexibility in choices regarding the types of elements that can be adjusted for the desired phase distributions. Furthermore, FSS's are in general flat, thin, low-weight, potentially low-cost and easy to built, and have shown to provide good focusing properties [e.g., 8]. On the other hand, the disadvantages of FSS's are that they may generally be narrow band and may also be sensitive to the angle of incidence [12].

In this paper, we present some of the results of our numerical analysis for focusing properties of a reflective lens that consists of two parallel gangbuster surfaces above a ground plane, with capability of allowing different focal points for two different polarization components. 


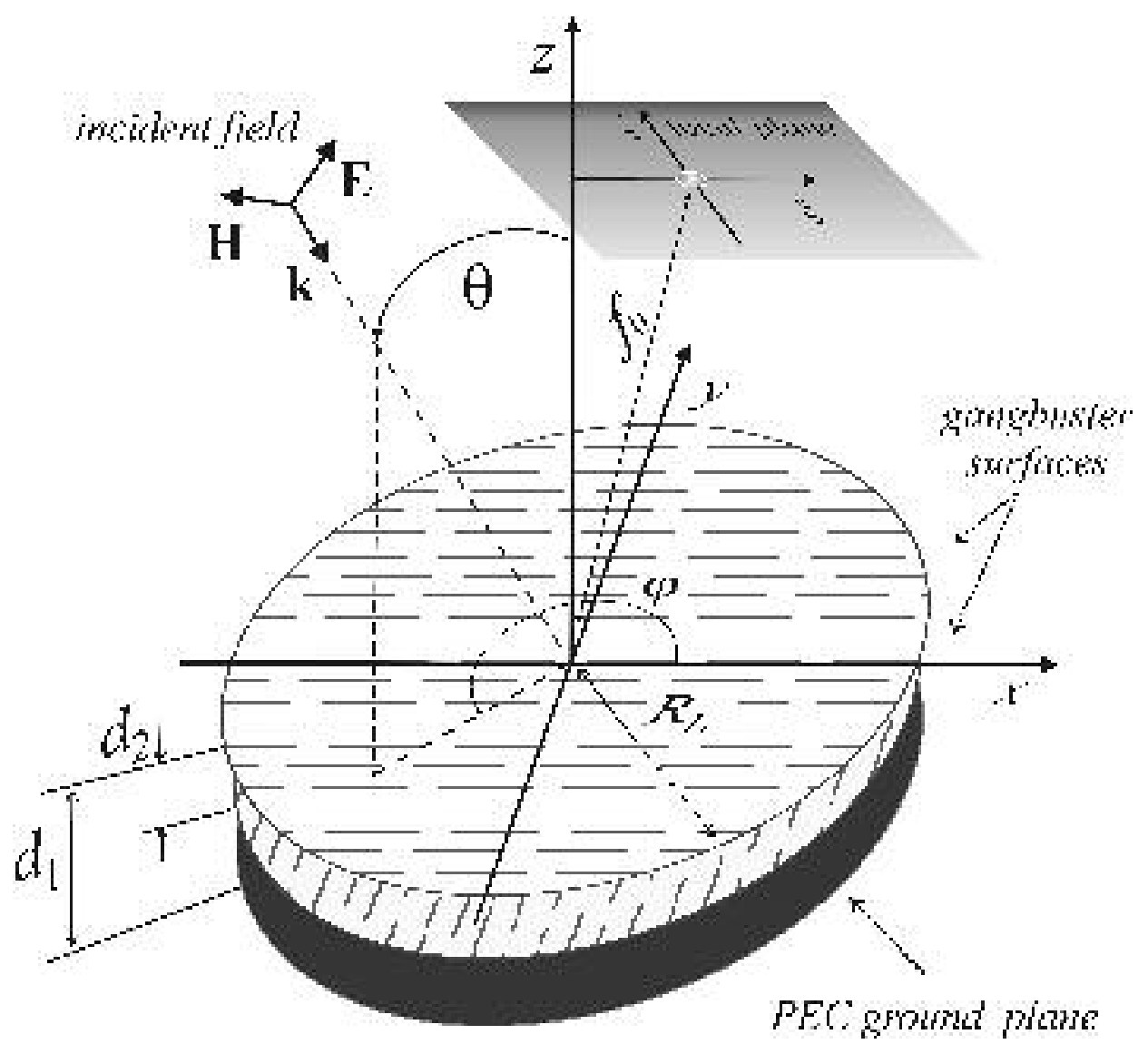

Fig. 1. Geometry of the problem of reflective lens. Bilayer gangbuster-surface (GS) reflective lens with surfaces at distances $d_{1}$ and $d_{2}$ above a perfectly electric conducting (PEC) ground plane. (Note: In our numerical analysis, the separation values $d_{1}$ and $d_{2}$ are taken to be electrically very small; however in the sketch shown in (a) they are drawn large in order to show orientation of wires in the bottom GS layer.)

\section{Geometry of a Bilayer Planar FSS Reflective Lens}

The two-layer planar FSS reflective lens system consists of two thin circular layers of radius $R_{L}$ of non-uniform gangbuster surfaces (GS) above the perfectly electric conducting (PEC) ground plane at distances $d_{1}$ and $d_{2}$, respectively, as shown in the Fig.1. The origin of Cartesian coordinate system coincides with the center of the upper layer. The non-uniform GS is presented in Fig. 2. This is a flat superdense array of 
parallel thin wires of finite length, each wire has a radius $a_{w}$ and certain length $l_{i j}$. The centers of the wires form a square lattice with grid spacing $D_{w}$, which is small compared with the wavelength of operation. The wires make the angle $\alpha_{w}$ with the lattice axes, where $\tan \alpha_{w}=1 / n_{w}, n_{w}$ is an integer, specifying the type of the gangbuster surface [12]. The length of the wires can be comparable with the wavelength, but spacing between them is much smaller than the wavelength. The wires of top GS layer are oriented orthogonal to the wires of the bottom GS layer.

Clearly when a plane wave is incident upon this structure, each GS layer interacts with the components of the electric field parallel with its wire constituents, and thus the electric field component orthogonal to the wire axis of a layer is effectively not "seen" by that layer. Since we have two GS layers with perpendicularly oriented wires, (Fig. 1), each layer interacts with a separate orthogonal polarization component of the incident wave. Therefore, this system of two GS layers together with the ground plane will reflect the incident wave totally, with specific reflection phase distribution that is dependent on the local wire length in each GS layer for each polarization. It is then possible to design each GS layer in order to focus the wave with a specific polarization at a given point in the focal space. Thus such a lens can segregate the two polarization components of the incident wave, focusing each at a separate focal point, or even at different focal planes. 


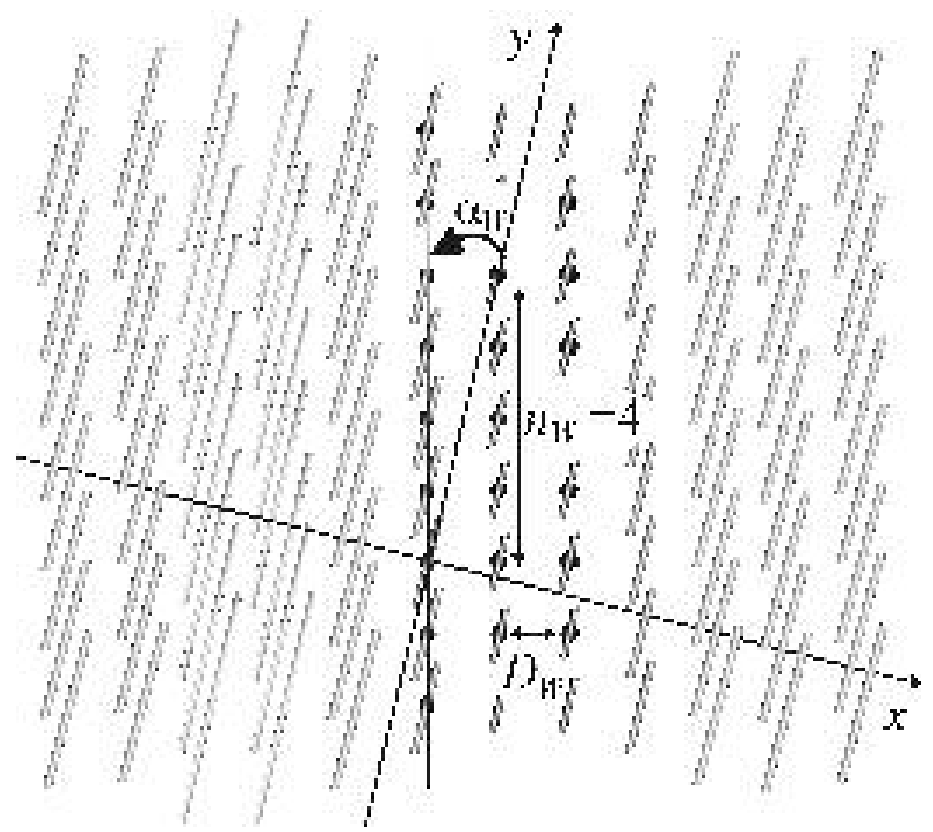

Fig. 2. A segment of the nonuniform GS layer, showing parallel wires with differing lengths.

\section{Design Lens Equation}

When this reflective surface is illuminated obliquely by a plane wave, in order for the reflected wave to be focused at a certain focal point in space, the phase difference between the reflected wave and the incident wave at any point $(x, y)$ on the lens surface should be as follows

$$
\Psi_{m}=k_{0}\left[-x \sin \theta \cos \varphi-y \sin \theta \sin \varphi+\left(\left(f_{x m}-x\right)^{2}+\left(f_{y m}-y\right)^{2}+f_{z m}^{2}\right)^{1 / 2}\right]
$$

where $x$ and $y$ are coordinates of any given point on the lens surface, $k_{0}=2 \pi / \lambda_{0}$ is the free-space wavenumber, $(\theta, \varphi)$ (shown in Fig. 1) indicate the direction of the incident wave in the spherical coordinate, and $\left\{f_{x m}, f_{y m}, f_{z m}\right\}$ are the Cartesian coordinates of the focal points desired for reflected wave from the $m^{\text {th }}$ GS layer. These values $\left\{f_{x m}, f_{y m}, f_{z m}\right\}$ can be chosen differently to separate two orthogonal polarizations. In 
order to design the lens surface using the GS layers, we take the incident wave to be incident obliquely with its wave vector in the $x z$ plane, i.e., the angles are assumed to be $\theta_{\text {design }}=45^{\circ}$ and $\varphi_{\text {design }}=270^{\circ}$. We then need to find the phase difference $\Psi_{m}$ at the center of the $i j^{\text {th }}$ wire on the $m^{\text {th }}$ layer surface $(m=1,2)$. This can be expressed as

$$
\Psi_{m}\left(x_{i j}, y_{i j}\right)=k_{0}\left[x_{i j} \sin \theta_{\text {design }}+\left(\left(f_{x m}-x_{i j}\right)^{2}+\left(f_{y m}-y_{i j}\right)^{2}+f_{z m}^{2}\right)^{1 / 2}\right]
$$

where $\left(x_{i j}, y_{i j}\right)$ are the coordinates of the center of wire $i j^{\text {th }}$. The length of the $i j^{t h}$ wire of the $m^{\text {th }}$ layer should then selected such that to achieve the phase shift $\Psi_{m}\left(x_{i j}, y_{i j}\right)$ given in (2). One way to obtain the proper wire lengths in each GS layer and to reconstruct such a GS that can provide the desired phase distribution of Eq. (2) is to find out how the phase of the reflection coefficient of an infinitely extent uniform GS layer above a perfectly conducting (PEC) ground plane depends on the wire length. Although the analysis for such an infinite case treats only the uniform periodic GS, this can be a reasonable approximation for the array of wires with only small local differences in wire lengths. An analogous approximation for the array of patches has already been used for the analysis of reflectarray antennas (see e.g., [9]). Utilizing the standard full-wave method of moment (MoM) technique used in [13-14], we first consider the scattering of an incident plane wave $\vec{E}^{i n c}$ from an infinite uniform periodic GS alone [12-14]. 


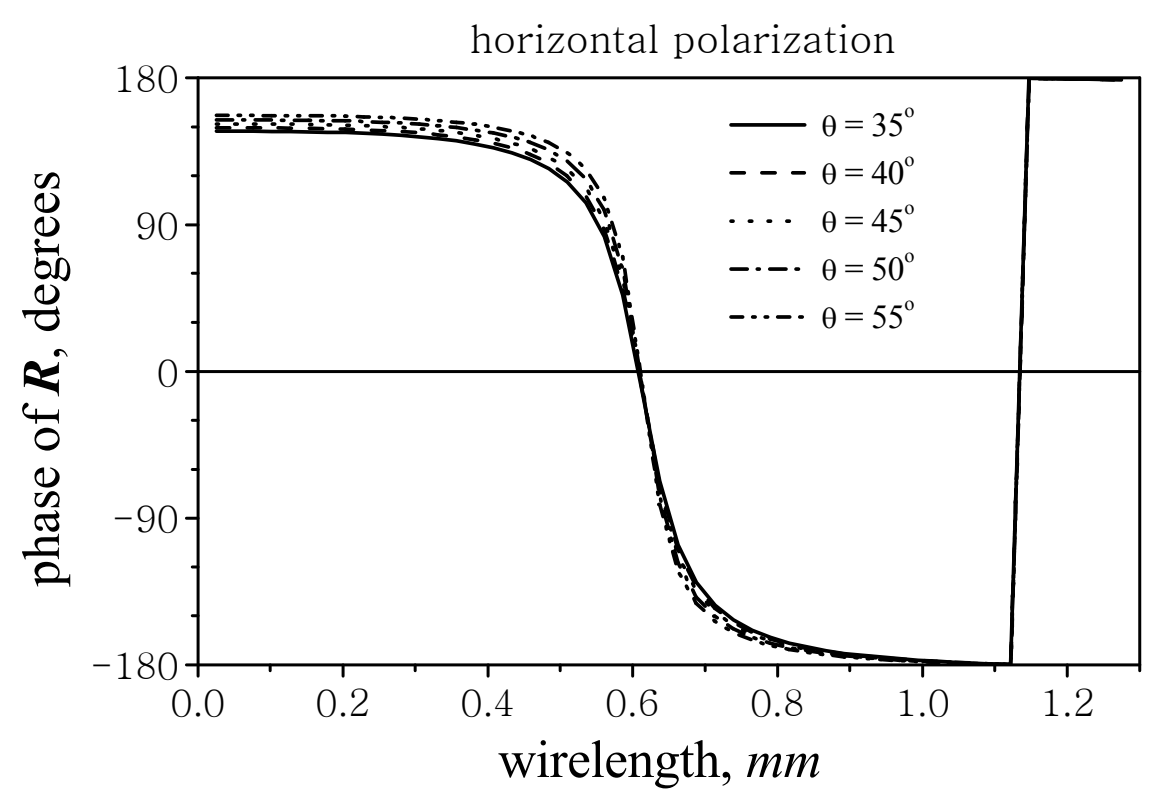

(a)

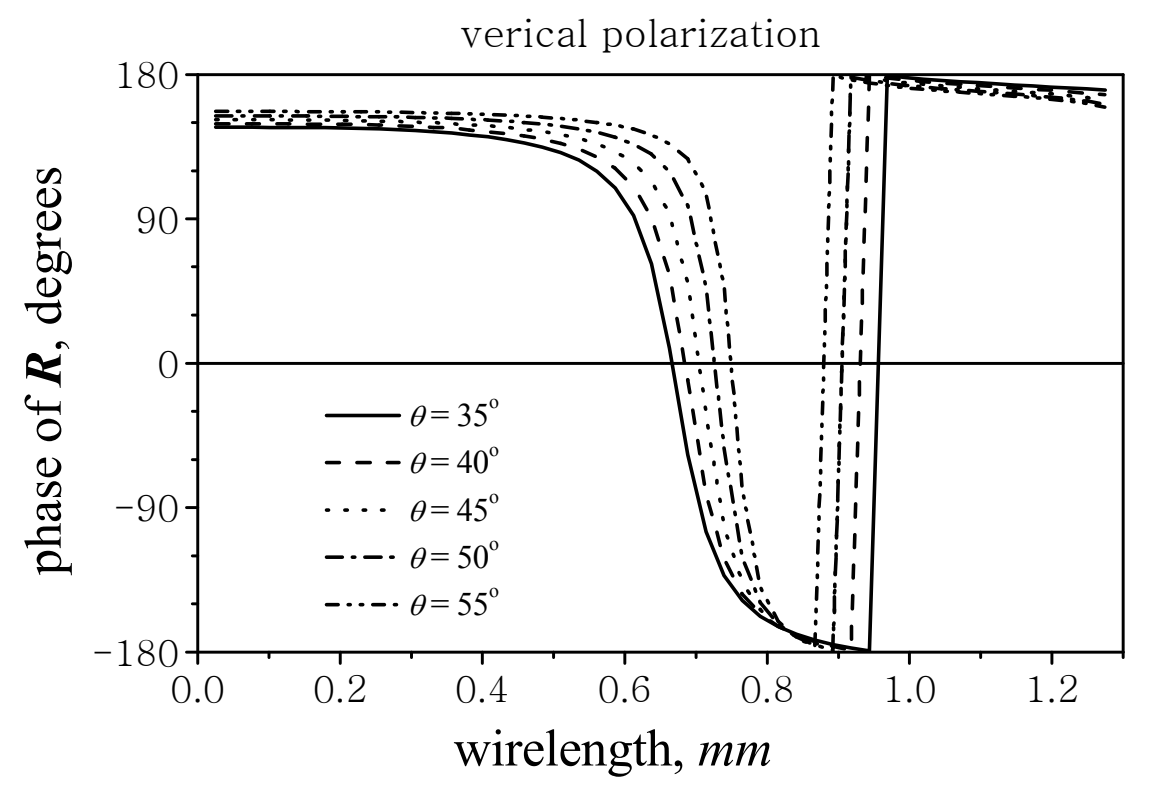

(b)

Fig. 3. Phase of the reflection coefficient for (a) horizontal polarization, (b) vertical polarization for the GS layer situated at the distance $d_{m}$ above a PEC ground plane versus the wire length, for various angles of incidence. $\lambda_{o}=1.8 \mathrm{~mm}, d_{m}=0.1 \mathrm{~mm}$, wires are parallel to $y$-axis. 
Then, by replacing the actual GS by an equivalent thin anisotropic impedance sheet as was done in [e.g., 13-14], we employ the transmission-line theory to determine the phase of the reflection coefficient $R$, as a function of the wire length of the uniform GS, for the wave incident on this GS when it is at a distance $d_{m}$ above an infinitely extent perfectly electric conducting (PEC) ground. As an example, Fig. 3 illustrates behavior of the phase of the reflection coefficient for a horizontally polarized wave (i.e., the electric vector being parallel with the wires on the GS layer) for various angles of incidence $\theta$ and $\varphi$ (see Fig. 1). All curves posses several basic features: First, as can be seen in Fig. 3, the phase largely depends on the length of wires. In the "middle" region the value of the phase changes considerably. Second, the phase variation does not cover the entire period $\left\{-180^{\circ}, 180^{\circ}\right\}$, since for small wire lengths, the gap in the initial phase is determined by the separation $d_{m}$. However, by varying the values of $n_{w}$ and $D_{w}$ of the GS layer, it is possible to reduce this gap to a value that is reasonably acceptable for design criteria. The scattering characteristics of the GS layer may be sensitive to the angles of incidence for a certain polarization (compare horizontal polarization (a) and vertical polarization (b) cases in Fig. 3), which may also provide some limitations in the lens application. When the lens is designed for one particular angle of incidence (usually the central/design angle), then as the angle of incidence differs considerably from the design angle, the wires on the GS layers may cause a different phase shift and, thus, a desired phase distribution on the lens may be distorted, and as a result, focusing deterioration may occur for angles of incidence other than the design angle. In all the results we present in this paper, we use the values $n_{w}=5, D_{w}=0.25 \mathrm{~mm}, a_{w}=0.001 \mathrm{~mm}$. Our numerical study has shown that if any type of GS can provide at least $80 \%$ of the phase compensation on the 
lens surface, by and large, the lens will focus the incident wave satisfactorily and may not differ drastically if we change $n_{w}$ of GS.

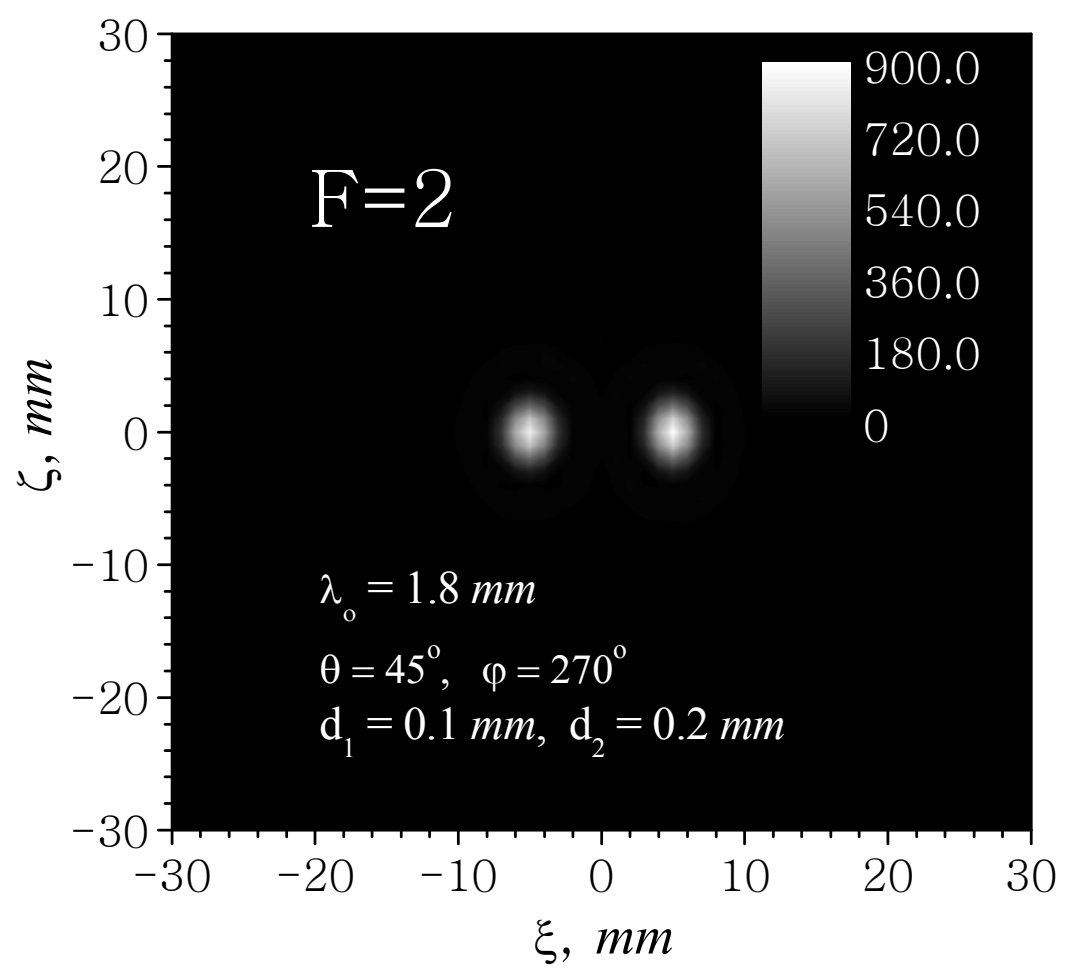

Fig. 4. Intensity of the electric field in the focal plane (Normalized with respect to the electric field intensity of the incident wave). $F / \#: 2, f_{y 1,2}=f_{z 1,2}=141.4 \mathrm{~mm}$, $f_{x 1}=-5 \mathrm{~mm}, f_{x 2}=5 \mathrm{~mm}, R_{L}=70 \mathrm{~mm}, f_{0}=200 \mathrm{~mm}$. The upper layer focuses $T E^{y}$ field components only at $f_{x 2}=5 \mathrm{~mm}$, and the lower layer focuses $T E^{x}$ fields at the focal point with $f_{x 1}=-5 \mathrm{~mm}$.

\section{Field Intensity in the Focal Plane-Numerical Results}

There are several well-known techniques one can use in order to estimate the fields at a certain point in space, when the field distribution over a closed boundary is known. Some of the commonly used methods are based on Surface Equivalence Theorem [15] or 
the Stratton-Chu formula [16]. In our analysis here, we use the surface equivalence theorem, in which actual fields on the surface $S$ surrounding the lens are replaced by equivalent electric and magnetic surface current densities $\vec{J}_{m}^{S}=\vec{n} \times \vec{H}_{m}$ and $\vec{M}_{m}^{S}=-\vec{n} \times \vec{E}_{m}$ on each GS layer, where $\vec{n}$ is the unit normal vector to the lens surface, i.e., along the $x$ axis, and $\vec{E}_{m}$ and $\vec{H}_{m}$ are total electromagnetic fields on the lens surfaces $m=1$ and $m=2$. From the knowledge of the equivalent current densities, one can use the method of auxiliary vector potentials, and find the electric and magnetic fields at any point in the space [15]. Here we present some of our numerical results for the distributions of the electric field intensity in the focal plane of the bilayer reflective lens, designed specifically to have two separate focal points for two orthogonal polarizations of the incident wave, at the coordinates $f_{y 1,2}=f_{z 1,2}=141.4 \mathrm{~mm}, f_{x 1}=-5 \mathrm{~mm}$ and $f_{x 2}=5 \mathrm{~mm}$. The $F$-number, which is given here as the ratio of the focal distance over the apparent aperture of the lens, is determined here for the reflective lens as $F=f_{0} /\left(2 R_{L} \cos \theta_{\text {design }}\right)$, where $f_{0}$ is the distance between focal point and the center of the upper GS layer, and $R_{L}$ is the radius of the lens. Fig. 4 and 5a show the field intensity at the focal plane, for the lenses designed primarily for the design angle, i.e., the angles of incidence are $\theta=45^{\circ}$ and $\varphi=270^{\circ}$. 


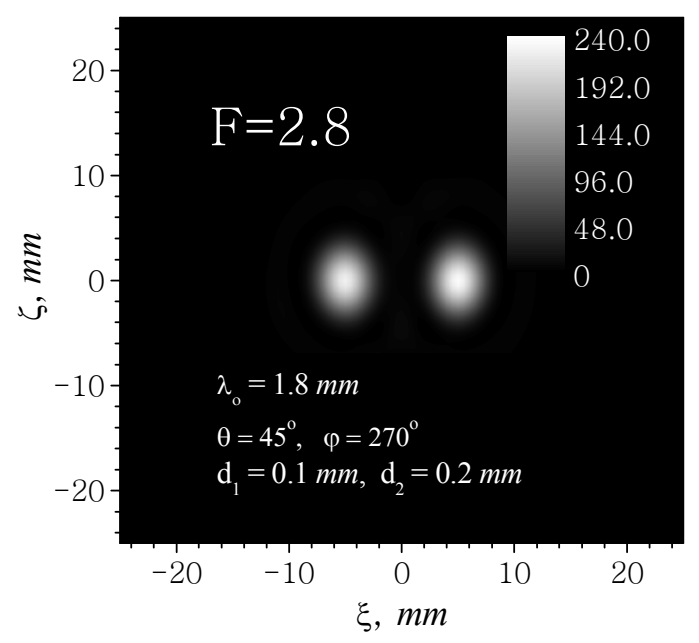

(a)

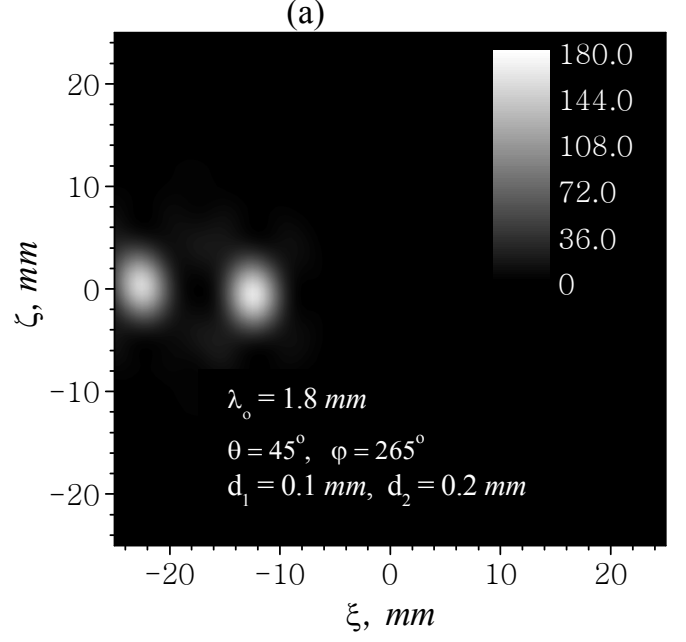

(c)

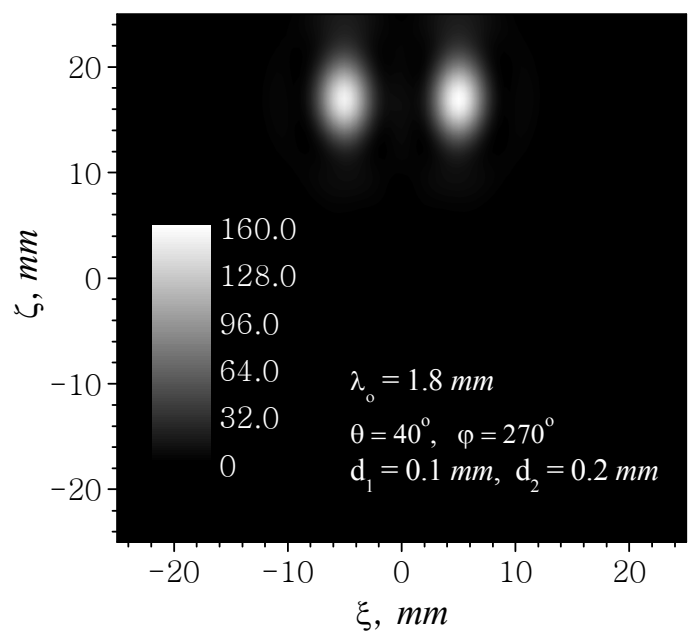

(b)

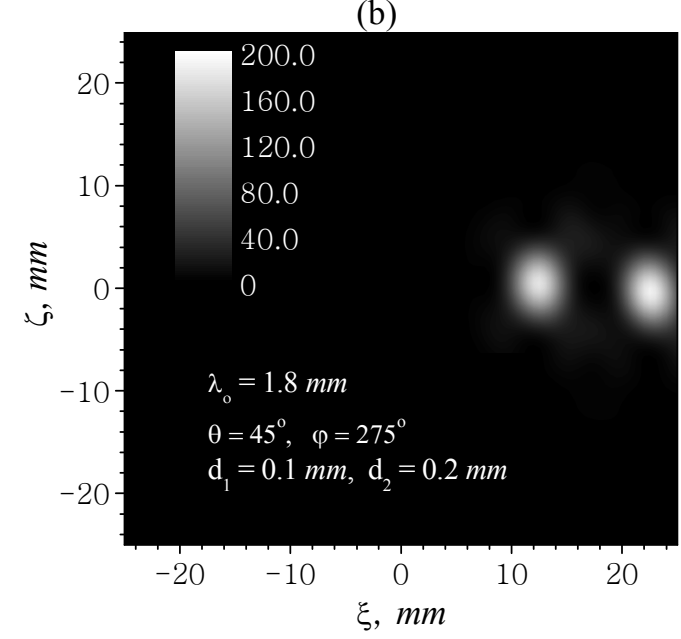

(d)

Fig. 5. Normalized intensity of the electric field in the focal plane for various angles of incidence.

$F / \#=2.8, f_{y 1,2}=f_{z 1,2}=141.4 \mathrm{~mm}, f_{x 1}=-5 \mathrm{~mm}, f_{x 2}=5 \mathrm{~mm}, R_{L}=50 \mathrm{~mm}, f_{0}=200 \mathrm{~mm}$

As can be seen, the focusing effect is very prominent, and the bigger lens (i.e., the lens with smaller F/\# shown in Fig. 4) causes higher peak intensity at the focal points, as expected. We note that these lenses operate as polarization splitters, focusing each polarization at a separate focal point, as they were designed to do so. If we change angles of incidence, the lens performance is still quite satisfactory as illustrated in Fig. 5b-d. The 
level of peak intensity is, however, lowered but focal separation for orthogonal polarizations effectively stays unchanged.

\section{Summary}

A polarization-dependent bi-focal two-layer FSS reflective lens on the base of gangbuster surfaces has been investigated and analyzed numerically. Such reflective lenses can be good candidates for polarization splitters in polarimetric imaging systems in millimeter wave regime. They are polarization-selective, and can be flat, thin, and low weight.

\section{Acknowledgements}

This work was supported in part by the U.S. Office of Naval Research (ONR), through grant N00014-02-1-0157.

\section{References}

[1] Z. Mei, W.B. Dou, "Analysis of focal field of small F-number imaging systems at millimeter wavelength," Journal of Electromagnetic Waves and Applications, Vol. 16, No. 6, 2002, pp. 837-849.

[2] J.A. Clarke, R.J. Dewey, "Millimeter wave imaging lens antenna," Int. journal of Infrared and Millimeter Waves, Vol. 5, No. 1, 1984, pp.91-100.

[3] Z. Mei, W.B. Dou, "Performance of hyperbolical and spherical lens imaging systems at millimeter wavelengths," Journal of Electromagnetic Waves and Applications, Vol. 16, No. 8, 2002, pp. 1077-1093.

[4] W.B. Dou, C. Wan, "An analysis of diffractive lenses at millimeter wavelength," Microwave and Optical Technology Letters, Vol. 11, No. 5, 1996, pp. 277-279.

[5] K. Hirayama, E.N. Glytsis, T.K. Gaylord, "Rigorous Analysis of Diffractive Cylindrical Lenses," J. Opt. Soc. Am. A., Vol. 13, No. 11, 1996, pp. 2219-2231. 
[6] Y.J. Guo, S.K. Barton, "Flat printed lens and reflector antennas," IEE Conference Publication, Proceedings of the 9th International Conference on Antennas and Propagation, v 1, n 407, Antennas, Eindhoven, Neth, 1995, p 253-256.

[7] D.M. Pozar, "Flat Lens Antenna Concept Using Aperture Coupled Microstrip Patches," Electronic Letters, Vol. 32, No. 23, 7th November 1996.

[8] S. Chandran, J.C. Vardaxoglou, "Experimental results on the focusing properties of tapered frequency-selective surfaces," Microwave and Optical Technology Letters, Vol. 11, No. 5, pp. 277-279, 1996.

[9] D.M. Pozar, T.A. Metzler, "Analysis of a Reflectarray Antenna Using Microstrip Patches of Variable Size,” Electronic Lett, Vol. 29, No. 8, pp. 657-658, 1993.

[10] J. A. Encinar, "Design of Two-Layer Printed Reflectarrays Using Patches of Variable Size," IEEE Trans. Antennas Propag., Vol. 49, No. 10, 2001, pp. 14031410 .

[11] J. A. Encinar and J. A. Zornoza, "Broadband Design of Three-Layer Printed Reflectarrays," IEEE Transactions on Antennas and Propagation, Vol. 51, No. 7, pp. 1662-1664, July 2003.

[12] B.A. Munk. Frequency Selective Surfaces: Theory and Design. A WileyInterscience Publication, New York, 2000.

[13] C.A. Moses. A Theoretical Study of Electromagnetic Feedforward/Feedback Media and Wire Media. PhD Dissertation, The University of Pennsylvania, 1997.

[14] C. A. Moses and N. Engheta, "Electromagnetic Wave Propagation in the Wire Medium: A Complex Medium with Long Thin Inclusions," in the Special Issue of Wave Motion on the topic of "Electrodynamics in Complex Environments", Vol. 34, No. 3, pp. 301-318, September 2001.

[15] C. A. Balanis, Advanced Engineering Electro-magnetics, Wiley, New York, 1989.

[16] J. A. Stratton, Electromagnetic Theory, McGrawHill, New York,1941 